OPEN ACCESS

Edited by:

Weili $\mathrm{Fu}$,

Sichuan University, China

Reviewed by:

Xiao Gong,

Wuhan University of Technology,

China

Wen Shi,

University of Nebraska Medical

Center, United States

${ }^{*}$ Correspondence:

Xinhua Qu

xinhua_qu@126.com

Zhifeng Yu

zfyu@outlook.com

${ }^{t}$ These authors have contributed equally to this work and share first authorship

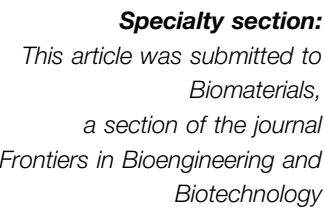

Received: 30 June 2021 Accepted: 27 September 2021

Published: 11 November 2021

Citation:

Du J, He Z, Cui J, LiH, Xu M, Zhang S, Zhang S, Yan M, Qu X and Yu Z (2021) Osteocyte Apoptosis Contributes to Cold Exposure-induced Bone Loss. Front. Bioeng. Biotechnol. 9:733582. doi: 10.3389/fbioe.2021.733582

\section{Osteocyte Apoptosis Contributes to Cold Exposure-induced Bone Loss}

\author{
Jingke $\mathrm{Du}^{1+}$, Zihao $\mathrm{He}^{2 \dagger}$, Junqi Cui ${ }^{3+}$, Hanjun $\mathrm{Li}^{1}$, Mingming $\mathrm{Xu}^{1}$, Shuhong Zhang ${ }^{1}$, \\ Shuangyan Zhang ${ }^{1}$, Mengning $\mathrm{Yan}^{1}$, Xinhua $\mathrm{Qu}^{4 *}$ and Zhifeng $\mathrm{Yu}^{1 *}$ \\ ${ }^{1}$ Shanghai Key Laboratory of Orthopedic Implants, Department of Orthopedic Surgery, Shanghai Ninth People's Hospital, \\ Shanghai Jiao Tong University School of Medicine, Shanghai, China, ${ }^{2}$ Arthritis Clinic and Research Center, Peking University \\ People's Hospital, Peking University, Beijing, China, ${ }^{3}$ Department of Pathology, Shanghai Ninth People's Hospital, Shanghai Jiao \\ Tong University School of Medicine, Shanghai, China, ${ }^{4}$ Department of Bone and Joint Surgery, Renji Hospital, School of \\ Medicine, Shanghai Jiao Tong University, Shanghai, China
}

Emerging evidence indicates that bone mass is regulated by systemic energy balance. Temperature variations have profound effects on energy metabolism in animals, which will affect bone remodeling. But the mechanism remains unclear. 2-month-old C57BL/6J male mice were exposed to cold $\left(4^{\circ} \mathrm{C}\right)$ and normal $\left(23^{\circ} \mathrm{C}\right)$ temperatures for 28 days and the effects of cold exposure on bone mass was investigated. Micro-computed tomography results showed that bone volume fraction was significantly reduced after 14 days of exposure to cold temperature, and it was recovered after 28 days. Ploton silver staining and immunohistochemical results further revealed that exposure to cold decreased canalicular length, number of E11-and MMP13-positive osteocytes after 14 days, but they returned to the baseline levels after 28 days, different from the normal temperature control group. In addition, change of Caspase-3 indicated that exposure to cold temperature augmented apoptosis of osteocytes. In vitro results confirmed the positive effect of brown adipocytes on osteocyte's dendrites and E11 expression. In conclusion, our findings indicate that cold exposure can influence bone mass in a time-dependent manner, with bone mass decreasing and recovering at 2 and 4 weeks respectively. The change of bone mass may be caused by the apoptosis osteocytes. Brown adipocyte tissue could influence bone remodeling through affecting osteocyte.

Keywords: cold exposure, bone mass, bone remodeling, osteocyte, perilacunar /canalicular remodeling

\section{INTRODUCTION}

Bone mass can be influenced by energy balance in many ways, and studies have demonstrated that caloric restriction and changes in leptin levels result in reduction of bone mass (Shi et al., 2008; Devlin et al., 2010). Likewise, the relationship between temperature and bone mass has aroused curiosity in recent years. It has been shown that low temperature has a negative influence on bone mass (Robbins et al., 2018), whereas warm temperature exposure $\left(34^{\circ} \mathrm{C}\right)$ prevents ovariectomies from bone loss through the microbiota in the intestinal tract (Chevalier et al., 2020). Further studies have revealed that cold temperature can activate the sympathetic nerves, which promote non-shivering thermogenesis of the muscle and have a deleterious effect on bone mineral density (Bonnet et al., 2007; Chen et al., 2019). Wee et al. have shown that exposure to cold decreases bone mass in the neuropeptide Y (NPY) wild-type mice, whereas the absence of NPY in the null mice obliterates these changes in bone mineral density (Wee et al., 2020). Steinberg et al. have found that the bone diameter and cortical thickness in the femoral midshaft decreased significantly after 69 days but increased after 
83 days of extreme cold $\left(5^{\circ} \mathrm{C}\right)$ exposure (Steinberg et al., 1981). Thus, determining the relationship between long-term exposure to low temperatures and bone mass will further meaningful insights about the phenomenon. Additionally, identifying the decisive factors that play critical role in this phenomenon will be of crucial significance in developing effective therapeutic strategies for skeletal or bone disorders.

Osteocytes are the most abundant cell type in the bone. Morphologically, they are stellate cells with many dendrites around them that function as important mechanical sensors (Dallas et al., 2013; Iolascon et al., 2013; Choy et al., 2020). In vivo, dendrites of osteocytes are buried in the bone canaliculus, through which osteocytes can connect with each other to exchange biological signals, mechanosensations, and absorb nourishment from the interstitial fluid (Wang et al., 2004; Bonewald, 2011; Wang, 2018). Interstitial fluid pressures in the bone lacuna-canalicular system vary with loads applied to the bone and cause deformation of the osteocyte membrane (Weinbaum et al., 1994). Therefore, the integrity of the canaliculus is important for the bone to maintain a standard mechanical response. It has been reported that osteocytes are associated with unload-induced bone loss in BCL transgenic mice (Moriishi et al., 2012; Komori, 2013). Osteocytes can cause resorption or deposition of bone matrix through perilacunar/ canalicular remodeling (Dole et al., 2020; Kegelman et al., 2020), which is another way for osteocytes to regulate bone mass. Reports have shown that decreased bone mineral density during lactation is mainly caused by perilacunar/canalicular remodeling (Qing et al., 2012; Wysolmerski, 2013). Mazur et al. have demonstrated that suppressed perilacunar/ canalicular remodeling is responsible for osteoarthritis (OA). Osteocytes inside the subchondral bone show a decreased length of the canaliculus at the end stage of osteoarthritis (Mazur et al., 2019). It has been found that YAP/TAZ deletion reduces bone mass and disturbs matrix collagen content and organization by suppressing osteocyte perilacunar/canalicular remodeling (Kegelman et al., 2020). In addition, systemic inhibition of TGF $\beta$ signaling induces poor bone quality through suppression of osteocyte perilacunar/canalicular remodeling (Dole et al., 2020). What's more, Eimear B. Dolan et al. found that thermal elevations $\left(47^{\circ} \mathrm{C}\right.$ for $\left.1 \mathrm{~min}\right)$ can cause osteocytes apoptosis and secretory function changing (Dolan et al., 2015). As a result, it is worth investigating whether there is a correlation between temperature and the canalicular system.

Enough osteocytes are important for maintaining the bone mass. Osteocyte apoptosis caused by aging (Almeida et al., 2007; Nicks et al., 2012), alterations in the mechanical environment (Bakker et al., 2004; Aguirre et al., 2006), fatigue-induced bone microdamage (Kennedy et al., 2012), or glucocorticoid levels (Heimann and Freiberger, 1960) can decrease bone mineral density. Furthermore, the fluctuating levels of RANKL, OPG, and VEGF play a significant role in this process. With the decrease in estrogen, bone loss is caused by osteocyte apoptosis-related osteoclast activities (Emerton et al., 2010). Osteocyte apoptosis induces a decline in OPG production and promotes the release of RANKL by adjacent osteocytes. As a result, bone turnover is accelerated, leading to a lower bone density (Jilka et al., 2013). Matrix metallopeptidase 13 (MMP13) is another important factor that affects bone mass. MMP13 is known to cleave collagen I in the extracellular matrix and potentially plays a role in the turnover of articular cartilage. Evidence suggests that MMP13 is related to bone quality and plays a significant role in osteocyte perilacunar remodeling (Mazur et al., 2019). As a transmembrane glycoprotein, E11/ podoplanin is vital for osteocyte differentiation, especially for the elongation of dendrites (Zhang et al., 2006). Deletion of E11 will impair the mechanical response of osteocytes and cause changes in bone mass (Staines et al., 2019; Qin et al., 2020). Accordingly, in this study, we evaluated the effects of cold-induced stress on bone mass and elucidated the potential underlying mechanisms.

\section{MATERIALS AND METHODS}

\section{In vivo Studies}

Two-month-old C57BL/6J male mice were purchased from Shanghai SLAC Laboratory Animal Co. (Shanghai, China), and the study was approved by the Animal Ethics Committee of Shanghai Ninth People's Hospital. Mice were fed commercial food and water under specific-pathogen free (SPF) conditions. For the in vivo study, 60 mice were haphazardly divided into two groups such as cold stimulation (cold) and room temperature (normal) groups (5 per cage); with 30 mice per group. In a nutshell, the mice in the cold group were grown at an incubator temperature of $4^{\circ} \mathrm{C}$, while the normal group was nursed in the same incubators at room temperature $\left(23^{\circ} \mathrm{C}\right.$ ) (Lim et al., 2012). At the end of each time point, mice were weighted and euthanasia with chloral hydrate, and then femurs and tibias were collected.

\section{Micro-computed Tomography Scanning}

After breeding under indicated conditions and for different periods, the femurs of the mice were fixed with $4 \%$ paraformaldehyde. Samples were scanned using micro-CT ( $\mu$ CT 80; Scanco, Zurich, Switzerland), as described previously (Zhou et al., 2019). The cancellous bone was selected at a distance of $1.9 \mathrm{~mm}$ from the femoral condyle and 100 layers from the distal end of the growth plate. The micro-CT parameters were as follows: voltage of $70 \mathrm{kV}$, electric current of $114 \mu \mathrm{A}$, and resolution of $10 \mu \mathrm{m}$ per pixel. The three-dimensional structural parameters studied included bone volume fraction (BV/TV), trabecular number (Tb.N), trabecular thickness (Tb.Th), and trabecular separation (Tb.Sp).

\section{Ploton Silver Staining}

After decalcification in 10\% EDTA for 3 weeks, all samples were embedded in paraffin. Sagittal sections of the medial compartment of the knee joint were cut at a thickness of $4 \mu \mathrm{m}$, followed by Ploton silver staining. Sections were deparaffinized and incubated in two steps: 50\% silver nitrate and $1 \%$ formic acid in $2 \%$ gelatin solution for $55 \mathrm{~min}$, as previously described. The stained slides were then washed in $5 \%$ sodium thiosulfate for $10 \mathrm{~min}$, dehydrated, cleared, and mounted. Consistent cortical regions were selected for evaluation in the 
medial and lateral areas of each specimen. Images were acquired at $100 \mathrm{x}$ magnification for the analysis (OLYMPUS, I $\times 71$ ). Quantification of the lacuno-canalicular area and canalicular length was quantified using ImageJ (Mazur et al., 2019).

\section{Immunohistochemical Staining}

All samples were decalcified in $10 \%$ ethylenediaminetetraacetic acid (EDTA) for 3 weeks and embedded in paraffin. For microstructure observation, 4 - $\mu$ m-thick sagittal sections of the medial compartment of the knee joint were cut, and immunohistochemical staining with MMP13 antibody (181651-AP, Proteintech, Wuhan, China, 1:50), caspase-3 (9,661, Cell Signaling Technology, Inc., Danvers, MA, United States, 1:1,000) antibody, RANKL (AF462, R and D Systems, Minneapolis, MN, United States, $10 \mu \mathrm{g} / \mathrm{ml}$ ) antibody, TRAP (ab191406, Abcam, 1: 100) and Osteocalcin (ab93876, Abcam, 1:100) antibody were performed. All the antibodies are diluted by $10 \%$ goat serum.

\section{In vitro Differentiation of BAT}

Brown adipocytes were isolated and cultured according to the above method (Ingram et al., 2017). Briefly, after euthanasia, the brown adipose tissue in the interscapular of 4-week-old C57BL/6 mice was collected, minced, and then digested with collagenase buffer (DMEM, $1 \mathrm{mg} / \mathrm{ml}$ collagenase I, 1\% fetal bovine Serum). The preadipocytes were filtered through a $70 \mu \mathrm{m}$ membrane and centrifuged, and then the preadipocytes were cultured with $10 \mathrm{ng} /$ $\mathrm{ml}$ bFGF (Pepro Tech), 10\% fetal bovine serum (Gibco) and pen/ strep (Life Technologies) in DMEM to a confluence of $80-90 \%$. The cells were passaged every 3 days. To get brown adipocytes, the cells were cultured with $10 \%$ fetal bovine serum (Gibco), $10 \mu \mathrm{g} / \mathrm{ml}$ insulin (Macklin), $1 \mu \mathrm{M}$ dexamethasone (Sigma), $0.5 \mathrm{mM}$ 3-isobutyl-1-methylxanthin, phosphodiesterase inhibitor (IBMX, Sigma), $5 \mu \mathrm{M}$ rosiglitazone (Sigma), $1 \mathrm{nM}$ T3 (Sigma) DMEM for 6 days, until brown adipocytes were formed.

\section{Preparation of BAT CM}

In order to obtain brown adipocytes conditioned medium (BAT $\mathrm{CM}$ ), brown adipocytes were cultured in DMEM containing $10 \%$ exosome free FBS and collected after $48 \mathrm{~h}$. Centrifuge at $3 \times 10^{2} \mathrm{~g}$ for $10 \mathrm{~min}$ to remove cells, and then centrifuge at $2 \times 10^{3} \mathrm{~g}$ for $10 \mathrm{~min}$ and $1 \times 10^{4} \mathrm{~g}$ for $30 \mathrm{~min}$ to remove cell debris and large vesicles (Cianciaruso et al., 2019; Song et al., 2019). The conditioned medium was filtered by $0.22 \mu \mathrm{m}$ and used for the cultivation of MLO-Y4.

\section{Immunofluorescence}

Osteocyte-like MLO-Y4 cells were used for studying osteocyte in vitro, which were kindly provided by Dr Lynda Bonewald (University of Missouri-Kansas City, Kansas City, MO). MLO-Y4 cells were cultured with $\alpha$-minimum essential medium ( $\alpha$-MEM; Hyclone) containing 5\% fetal bovine serum (FBS; Gibco), 5\% calf bovine serum (CBS; Gibco), and $1 \%$ penicillin/streptomycin (Sigma-Aldrich, St. Louis, MO) on rat tail collagen type I-coated (Solar bio, Beijing, China) dish. The experiment design included two groups: control group (Ctrl) and conditional medium group $(\mathrm{CM})$. MLO-Y4 cells $\left(1 \times 10^{5}\right)$ were seeded on $3.5 \mathrm{~cm}$ dishes coated with type I rat tail collagen
(Corning). The medium was changed when the cell density reached 50\%. Control groups continued to be cultured in the growth medium, while CM groups were cultured with brown adipocyte conditional medium and growth medium 1:1. After $24 \mathrm{~h}$, the cells were fixed with $4 \%$ paraformaldehyde (PFA) for 30 min, washed with PBS and then gradient incubated in $0.03 \%$ Triton X-100 and blocking buffer ( $1 \times$ TBST, $10 \%$ normal goat serum) for $1 \mathrm{~h}$ at room temperature $\left(23{ }^{\circ} \mathrm{C}\right)$. E11 antibody (Abcam, ab11936, 1:200) was added to the wells and overnight at $4^{\circ} \mathrm{C}$. After incubation with secondary antibody for $1 \mathrm{~h}$, TRITCphalloidin (Kingmorn, China) and DAPI (Thermo Scientific, US) were used for cytoskeleton and nuclear staining, then cells were observed and captured with confocal fluorescence microscopy.

\section{Quantitative reverse-transcription Polymerase Chain Reaction (qRT-PCR)}

Total RNA was extracted with TRIZOL reagent (Thermo Scientific, United States). After measuring the concentration, Quant script RT Kit (Promega, Madison, WI, United States) was used to convert total RNA into complementary DNA. A $10 \mathrm{ul}$ PCR reaction system composed of cDNA and SYBR Premix Ex Taq Mix (Selleck) was used to detect the expression level of messenger RNA (mRNA) in the Real-Time PCR system (Light Cycler 2.0; Roche Diagnostics GmbH, Mannheim, Germany). The primer sequences are shown in Table 1.

\section{Statistical Analysis}

Statistical analyses of data were performed using GraphPad Prism 5.0 (GraphPad Software Inc., San Diego, CA, United States). Each group have at least five mice. All quantitative values are presented as the mean \pm standard deviation, and differences were evaluated by $t$-test or two-way ANOVA, followed by Bonferroni correction for multiple comparisons. Statistical significance was set at $p<0.05$.

\section{RESULTS}

\section{Exposure to Cold Temperature Results in Bone Mass Variations}

To determine the effects of cold-induced stress on bone mass, the $\mathrm{C} 57 \mathrm{BL} / 6 \mathrm{~J}$ male mice were exposed to cold $\left(4^{\circ} \mathrm{C}\right)$ or room $\left(23^{\circ} \mathrm{C}\right)$ temperature for different time intervals (Figure 1A). Their bone mass parameters were calculated using micro-CT (Figure 1B). As shown in Figure 1C, the bone volume fraction $(\mathrm{BV} / \mathrm{TV})$ of the mice belonging to the cold-stress group reduced in 14 days compared to that of mice of the room temperature/ control groups; BV/TV same recovered after 28 days. Further, the trabecular thickness (Tb.Th) was found to be lower at 14 days in the mice belonging to the cold-stress group than in the control group, which is consistent with the increased trabecular separation (Tb.Sp). The mice were weighted at different time points. As shown in Figure 1D, there was no significant change in the body weight of the mice over time. These results indicate that stimulation with cold temperature has a negative effect on the bone mass after short-term cold 
TABLE 1 | Primer sequences for the quantitative reverse-transcription polymerase chain reaction.

\begin{tabular}{|c|c|c|}
\hline Target genes & Forward $\left(5^{\prime}-3^{\prime}\right)$ & Reverse $\left(5^{\prime}-3^{\prime}\right)$ \\
\hline Gapdh & AGGTCGGTGTGAACGGATाTG & TGTAGACCATGTAGTTGAGGTCA \\
\hline E11 & ACCGTGCCAGTGTTGTTCTG & AGCACCTGTGGTTGTTATITGT \\
\hline Ocn & CTGACCTCACAGATCCCAAGC & TGGTCTGATAGCTCGTCACAAG \\
\hline Sost & AGCCTTCAGGAATGATGCCAC & CTITGGCGTCATAGGGATGGT \\
\hline Runx2 & CCGGGAATGATGAGAACTA & ACCGTCCACTGTCACTTT \\
\hline
\end{tabular}
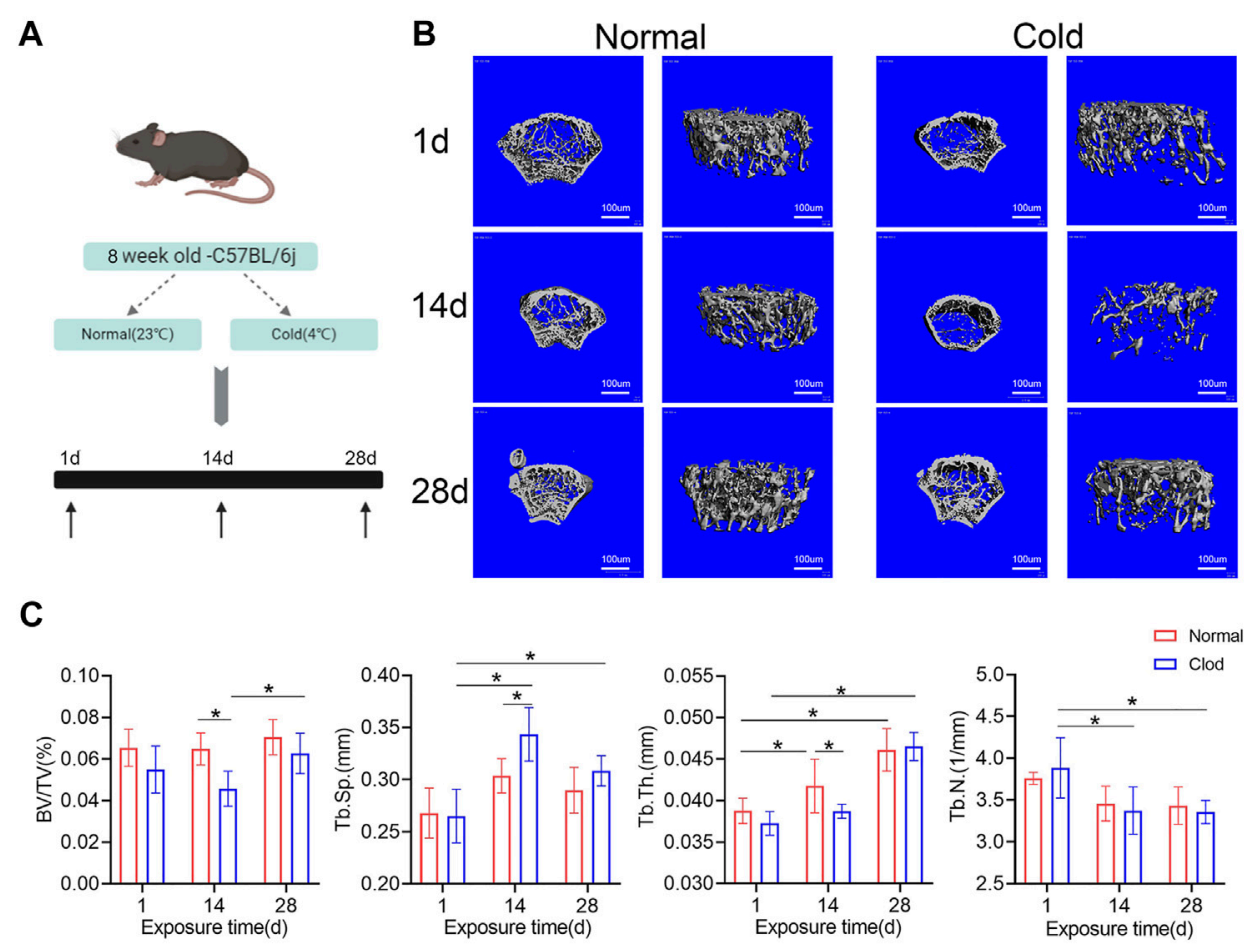

FIGURE 1 | Influence of cold-stress on bone mass in a time-dependent manner. (A) Schematic flow of the experimental design. Mice were nursed at different temperatures $\left(4\right.$ and $23^{\circ} \mathrm{C}$ ) and sacrificed after 1, 14 or 28 days. (B) The representative micro-CT 3D reconstruction images of different groups. (C) Trabecular bone microarchitecture of femurs showing bone volume/total volume (BV/TV), trabecular number (Tb.N), trabecular separation (Tb.Sp), and trabecular thickness (Tb. Th). Data are shown as mean $\pm \mathrm{SD}\left(\mathrm{n}=5\right.$ per group). Significance ( $p$ value) is calculated using two-way ANOVA, ${ }^{\star} p<0.05 ;$ Abbreviations: 1 day (1d), 14 days (14d), 28 days (28d), Normal: mice in the room temperature, Cold: mice in the $4^{\circ} \mathrm{C}$.

exposure (14 days), but with prolonged exposure, this effect gets debilitated. These findings suggest the existence of an adaptation process in bones that gets activated upon exposure to cold temperatures.

\section{Osteocyte Lacuna-Canalicular System Exhibits Variation During Exposure to Cold Temperature}

To study the effect of cold-induced stress on the lacunacanalicular system, the C57BL/6J mice were exposed to cold $\left(4^{\circ} \mathrm{C}\right)$ or room $\left(23^{\circ} \mathrm{C}\right)$ temperature for different time intervals and the bone lacunar-canalicular system was studied using the ploton silver staining technique as shown in Figure 2A. The lacuno-canalicular length and area were counted and are shown in Figures 2B,C. The results revealed that after 14 days of cold stimulation, canalicular area and length in the femur were reduced compared to that in control group mice. Further, the lacuno-canalicular area was enlarged and the canalicular length was recovered after 28 days of exposure (Figures 2B,C). E11, which was also known as podoplanin, is a cell membrane protein that can expressed in osteocytes (Nose et al., 1990; Schacht et al., 2003). As shown in Figure 2D,E, E11-positive osteocytes number reduced in the 14 days of the cold treated group, which consisted with the results of ploton silver staining. The lacuno-canalicular system is an important component of osteocytes and is a mechanical sensor that indirectly affects bone mass. The changes that occur during the cold-exposure treatment suggest that the alteration in bone mass may be caused by the variation in mechanical response ability. Decreased canalicular length at 14 days was consistent with the lower bone mass in the cold group unlike in the control group mice. Furthermore, prolonged 

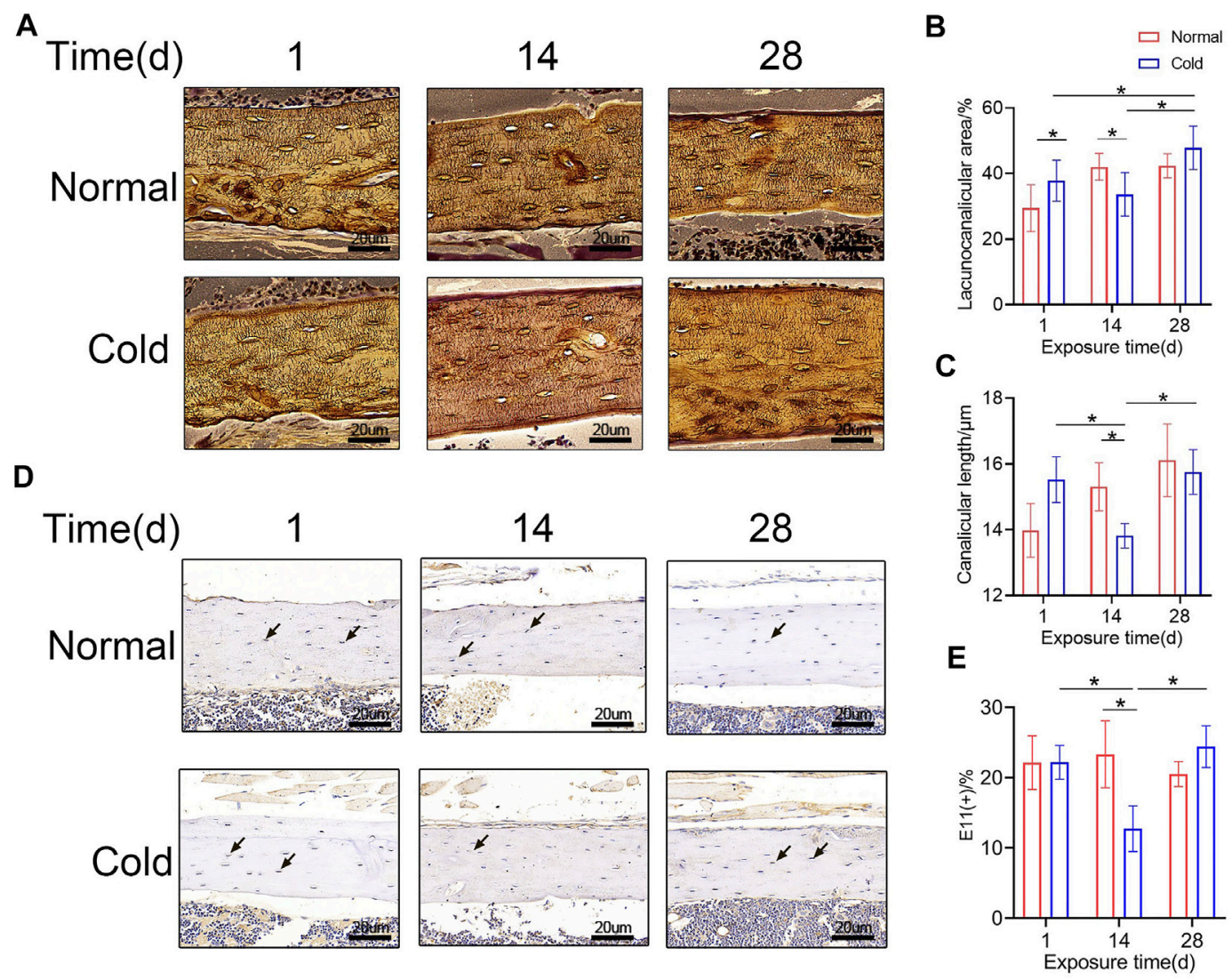

FIGURE 2 | Osteocyte canaliculus shows alterations with exposure to cold temperature. (A) Ploton silver stain was performed to show the canalicular network in cortical bone. (B-C) Lacuno-canalicular area and canalicular length were measured and the differences among the groups are shown. (D) Immunohistochemical stain of E11 in the femurs was performed. (E) E11-positive osteocytes are calculated and statistic results are shown. Black arrows: E11 positive osteocytes. Data are shown as mean \pm SD ( $n=5$ per group). Significance ( $p$ value) is calculated using two-way ANOVA, ${ }^{*} p<0.05$.

exposure to cold results in the recovery of bone mass, with elongation and enlargement of the lacuna-canalicular area.

\section{Cold Exposure Causes Osteocytes Apoptosis}

To study the effect of cold-stress on the fate of osteocytes in coldand room temperature-treated mice, $\mathrm{H}$ and $\mathrm{E}$ staining and immunohistochemical studies were performed. The $\mathrm{H}$ and $\mathrm{E}$ staining was employed to visualize the empty bone lacuna (Figure 3A). The results revealed that in the cold group, the proportion of empty lacunae increased on days 1 and 14 (Figure 3B). The immunohistochemical analysis was performed to determine the expression level of Caspase 3 (Figure 3C) in vivo. As the expression of Caspase3 is positively correlated with apoptosis, Caspase3-positive osteocytes were considered apoptotic. Figure 3D shows that the expression level of Caspase 3 in the cold-stress group was found to be augmented at 14 days compared to that in the control group, and there was no significant difference between the two groups after 28 days of exposure. Besides, immunohistochemical analysis was performed to determine the expression level of
MMP13 (Figure 3E) in vivo in both the groups of mice. As shown in Figure 3F, MMP13-positive osteocytes numbers decreased after 1 and 14 days in the cold environment. Changes in the empty lacuna, the number of Caspase3-and MMP13 positive osteocytes caused by the different temperatures indicate that exposure to cold can influence bone mass through osteocytes.

\section{Cold Affects the Secretory Function of Osteocytes}

As mentioned previously, cold exposure induced osteocyte apoptosis at 14 days. Reports show that dead osteocytes exhibit higher RANKL expression in the neighboring cells. To explore if there was a change in the level of RANKL, immunohistochemical staining was performed in the mice belonging to the cold/control groups, and representative pictures are shown in Figure 4A. Unsurprisingly, the proportion of RANKL-positive osteocytes was increased in the cold-stimulated group (Figure 4B), which is consistent with the variation trend of apoptotic osteocytes. Immunohistochemical staining were performed to show 


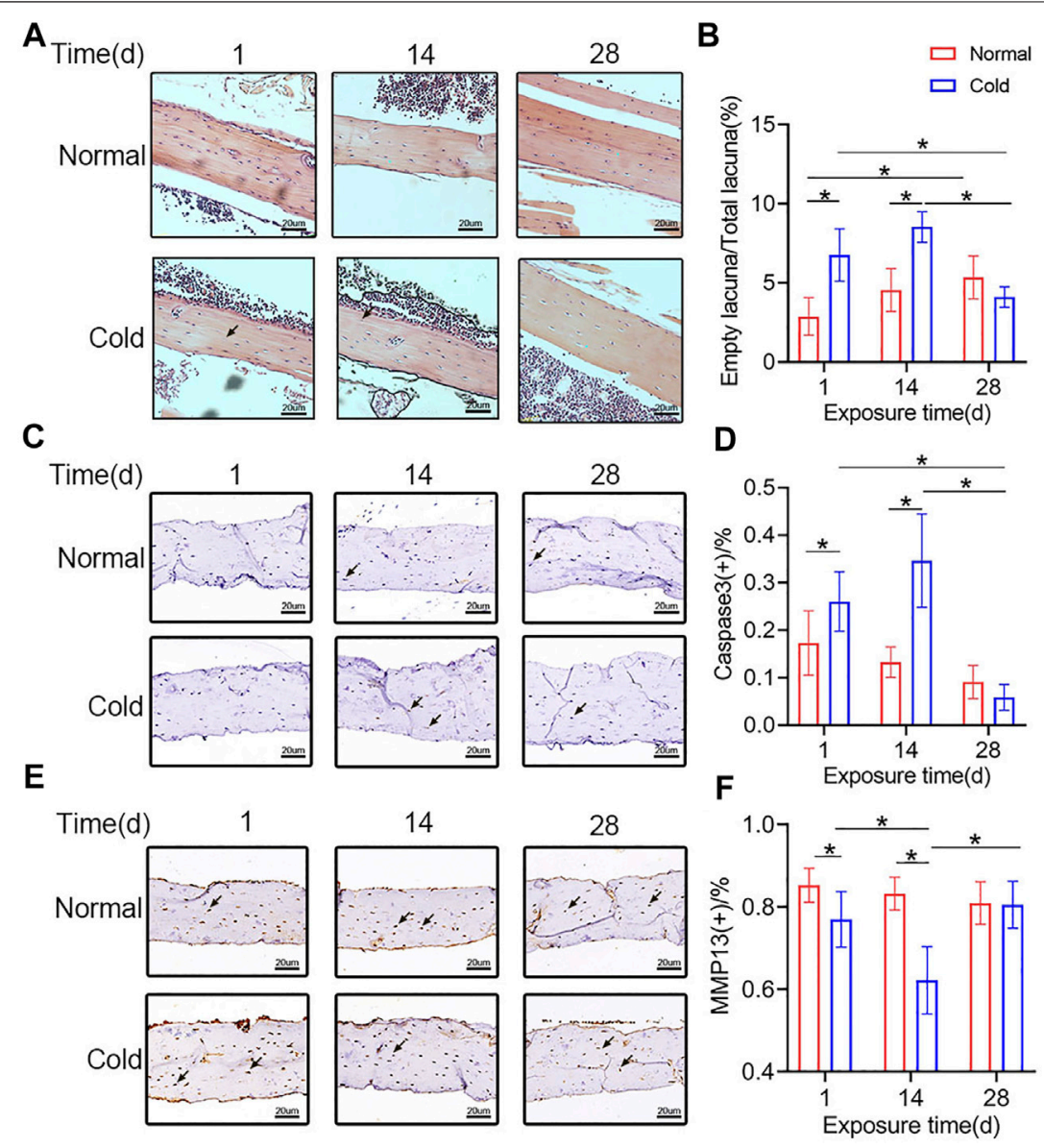

FIGURE 3 | Cold exposure induces osteocytes apoptosis. (A) Representative pictures of H and E staining in the femurs. (B) Quantification of empty lacunae in the Panel A. (C) Immunohistochemical stain of Caspase3 in the femurs was performed. (D) Caspase3-positive osteocytes are calculated and statistically represented. Black arrows: Empty lacuna or Caspase3 positive osteocytes. (E) Immunohistochemical stain of MMP13 in the femurs was performed. (F) MMP13-positive osteocytes are calculated and statistic results are shown. Black arrows: MMP13 positive osteocytes. Data are shown as mean \pm SD ( $n=5$ per group). Significance ( $p$ value) is calculated using two-way ANOVA, ${ }^{*} p<0.05$.

TRAP and osteocalcin (OCN) positive cells in bone tissue. As shown in Figure 4C and Figure 4D, TRAP positive osteoclasts and Oc. N/Tb.L were calculated, the results indicated that Oc. $\mathrm{N} / \mathrm{Tb}$.L were increased after 14 days in cold environment, which may explain the bone loss in the same time point. What's more, OCN positive osteocytes were decreased with the stimulation of cold exposure in the 14 and 28 days (Figures 4E,F). All of this makes it even more convincing that temperature plays a role in bone remodeling, and that's probably initiated by its effect on osteocytes.

\section{BAT CM Increases the Length of Osteocyte's Dendrites}

BAT CM was added to the culture medium of MLO-Y4, and immunofluorescent staining was performed to show the morphology of MLO-Y4 (Figure 5A). As shown in Figure 5B, dendrites of MLO-Y4 in the BAT CM group were longer than Ctrl group. Besides, relative mRNA expression levels of E11, Sost, and Ocn were promoted by BAT CM(Figure $5 \mathrm{C})$.

\section{DISCUSSION}

After exposure to the cold environment, the C57BL/6J mice showed a reduction in bone mass at 14 days as compared to the control conditions, but the changes were recovered at 28 days. Further studies demonstrated that there was an increased apoptotic osteocyte, shorter canalicular length, increased osteoclasts and changed RANKL and OCN expression level at 14 and 28 days in the cold group, which may be the important factors inducing undulation of bone mineral density.

The relationship between temperature and bone mass has fascinated scientists in the past few decades. It has been reported that nursing at $22^{\circ} \mathrm{C}$ can induce bone loss in C57BL/6J and C3H/ HeJ mice (Iwaniec et al., 2016; Robbins et al., 2018). Environmental factors may play a significant role in the energy metabolism of laboratory animals (Bektas et al., 2018). The reasons that have been considered are the reduced blood flow in the hind limb and changed volume of brown adipose tissue. Serrat et al. have attributed the shorter hind limb in a cold environment to cell proliferation and matrix production in 


\section{A}

Time(d)

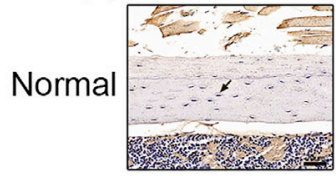

Cold

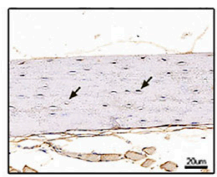

C Time(d)
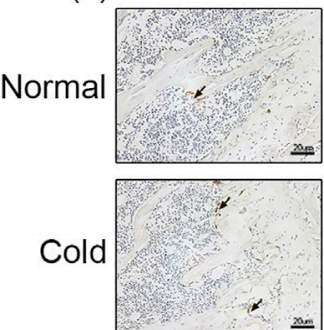

E
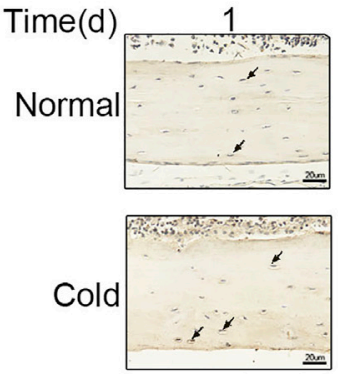

14

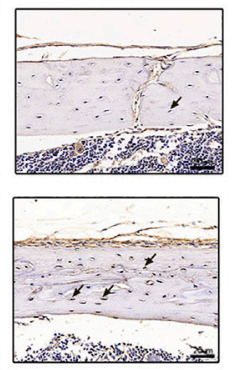

14

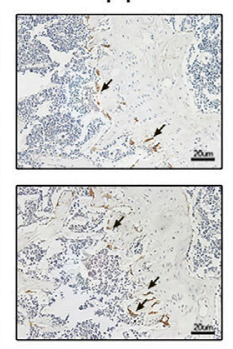

14
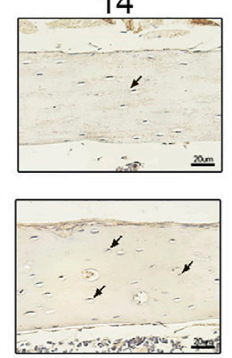

28

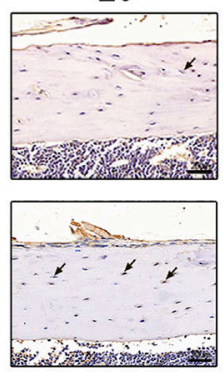

28

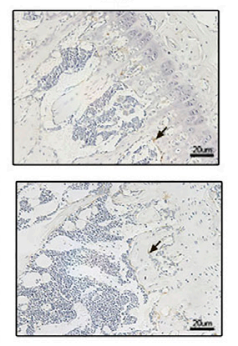

28

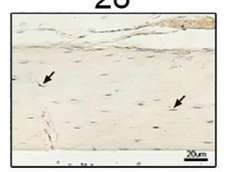

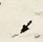

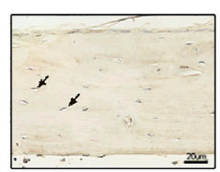

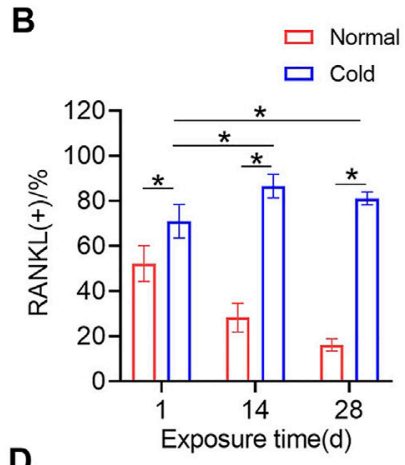

D

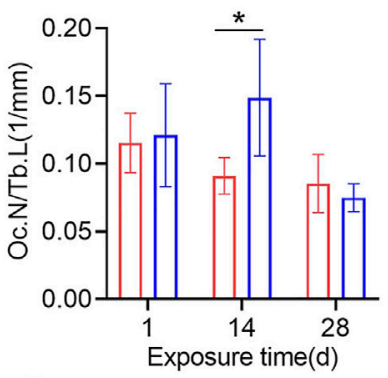

F

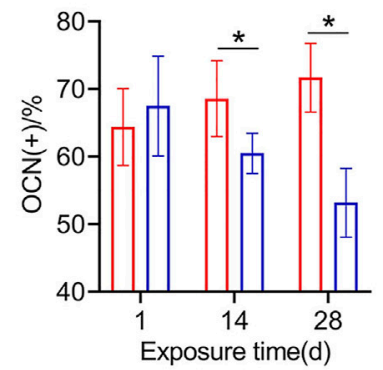

FIGURE 4 | Cold affects the secretory function of osteocytes (A) Immunohistochemical staining for RANKL in the femurs was performed. (B) RANKL-positive osteocytes were calculated and are statistically represented. (C-D) Immunohistochemical staining for TRAP in the femurs was performed and Oc. N/Tb.L were calculated. (E-F) Immunohistochemical staining for OCN in the femurs was performed and OCN positive osteocytes were calculated. Black arrows: RANKL-, TRAP-, or OCN-positive osteocytes. Data are shown as the mean $\pm \operatorname{SD}(n=5)$. Significance ( $p$ value) was calculated using two-way ANOVA, ${ }^{*} p<0.05$.

cartilage (Serrat et al., 2008). Animals in warmer places have longer limbs and stable bone mineral density (Serrat et al., 2008; Chevalier et al., 2020). In our study, when 2-month-old male C57BL/6J mice were exposed to $4^{\circ} \mathrm{C}$ for 14 days, bone loss was observed. However, when cold stimulation was prolonged to 28 days, the bone mass was partly recovered. Consistently, there have been reports that mice housed at $22^{\circ} \mathrm{C}$ for 9 weeks show lower BV/TV than thermoneutrality. Although $22^{\circ} \mathrm{C}$ is much higher than $4^{\circ} \mathrm{C}$, both are relatively low temperatures compared with room temperature. As bone mass showed no fluctuation in Robbins's study, which may be attributed by the difference of observation interval (Robbins et al., 2018).

To illustrate the potential mechanism, osteocyte canalicular networks were stained with ploton silver, and the statistical results showed shorter canalicular length in the cold group after 14 days of cold exposure as compared to control temperature. Immunohistochemical analysis was performed to test the E11 and MMP13 expression in vivo, and the results showed that short-term cold exposure was negatively related to E11 and MMP13 expression. As E11 was mainly expressed in the cell membrane of osteocytes, which has a positive effect on bone mass (Nose et al., 1990; Zhang et al., 2006), it is reasonable to speculate that cold can cause bone loss by affecting the expression of E11. Further, with the enlargement of brown adipose, restored E11 expression levels lead to recovery of bone mass (Wetterwald et al., 1996; Hadjiargyrou et al., 2001; Jung et al., 2019). Osteocytes can regulate bone mass through their lacuno-canalicular networks. They can secrete numerous factors, such as cathepsin $\mathrm{K}$ (CatK), prostaglandin, and matrix metalloproteinases (MMPs), and participate in perilacunar/ canalicular remodeling processes (Bonewald, 2011; Qing et al., 2012; Tang et al., 2012; Mazur et al., 2019). Prior studies have noted the importance of perilacunar/canalicular remodeling (PLR) in osteoarthritis (Mazur et al., 2019). As mentioned above, the lacuno-canalicular network is the mechanical sensory component of the bone. Changes in the length and 

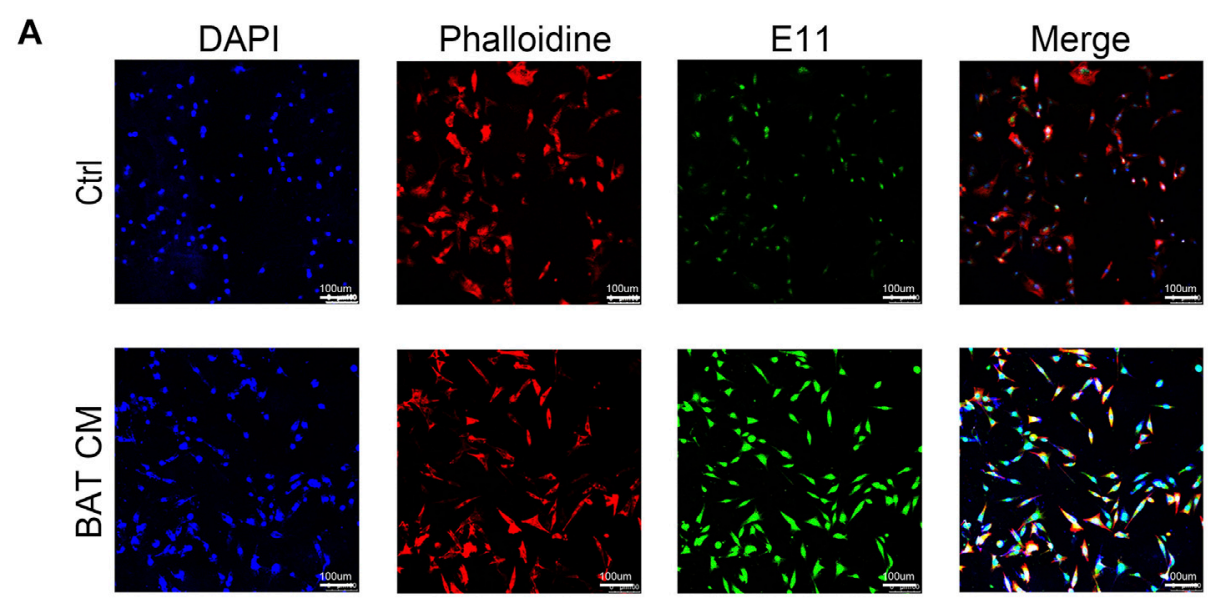

B

C

E11
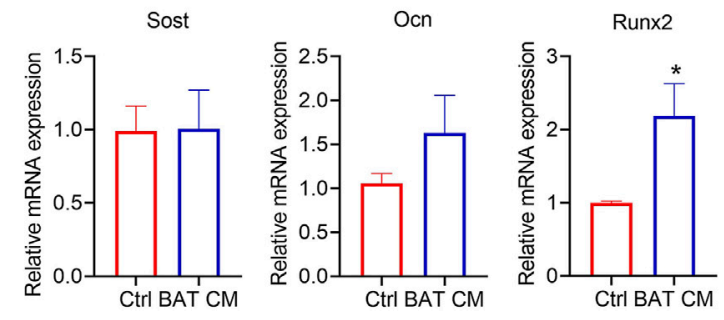

FIGURE 5 | BAT CM increase the length of osteocyte'sdendrites. (A,B) Immunofluorescent staining was performed to show the morphology of MLO-Y4 and dendrites length are calculated. (C) Relative mRNA expression levels of E11, Sost, and Ocn were detected by RT-PCR. Data are shown as mean \pm SD ( $\mathrm{n} \geq 3$ per group). Significance ( $p$ value) is calculated using student's t-test, ${ }^{\star} p<0.05$; Abbreviations: 1 day $(1 \mathrm{~d}), 14$ days (14d), 28 days (28d), Normal: mice in the room temperature ( $\left.23^{\circ} \mathrm{C}\right)$, Cold: mice in the $4^{\circ} \mathrm{C}$.

area disturb the metabolic balance of bone. During the lactation period, osteocytes have shorter dendrites and reduced bone mineral density (Wysolmerski, 2013). Mice in the $4^{\circ} \mathrm{C}$ environment had shorter canaliculus and smaller canalicular space as compared to the control conditions, which may partially explain the lower bone mass at 14 days.

$\mathrm{H}$ and $\mathrm{E}$ staining showed an increased number of empty lacunae after cold stimulation, which may be caused by osteocyte apoptosis. To determine whether cold-stress can influence osteocyte viability, Caspase- 3 positive cells in the cortical bone were counted and it was found that the number of apoptotic osteocytes in the cold group was higher than that in the normal group. As the initiator of bone remodeling, the osteocyte number is positively related to bone mass and apoptosis of which results in bone loss. The relationship between apoptotic osteocytes and bone mass has been well studied; osteoclastogenic cytokines released by apoptotic osteocytes activate osteoclasts and lead to bone resorption (Jilka et al., 2013). Aging, hormones, glucocorticoids, and mechanical stimulation have been found to play a critical role in osteocyte apoptosis (Bakker et al., 2004; Almeida et al., 2007; Jilka et al., 2013; Silva et al., 2020). Therefore, it is reasonable to assume that extremely low temperatures initiate the apoptosis of osteocytes and active osteoclasts. As one of the major cytokines secreted by osteocytes (Nakashima et al., 2011), RANKL is significantly increased in the cold as compared to the control group. RANKL can be elevated in many ways. Increased TNF- $\alpha$ and IL- 6 expression levels in the circulatory system promote the formation of RANKL, which directly activates osteoclasts ( $\mathrm{Wu}$ et al., 2017; Marahleh et al., 2019). Additionally, mechanical loading regulates the expression of RANKL by promoting the release of PGE2 (Iolascon et al., 2013; Uda et al., 2017). Apoptotic osteocytes facilitate the production of RANKL by neighboring cells (Hughes et al., 2020). In our study, RANKL was elevated in the cold group, which partly explains the decrease in bone mineral density. Research have shown that bone remodeling can be affected by temperature (Iwaniec et al., 2016; Ziętak et al., 2016), which may cause changed osteoclast number and osteoblast activity. Osteoclasts, the TRAP positive cell type and mainly causing bone resorption, were increased by cold stimulation in the 14 days. As a vitamin K-dependent protein, OCN seems to promote the osteoblast-to-osteocyte transition and also limit osteoclastogenesis (Atkins et al., 2009; Palermo et al., 2017). With the lengthening of cold stimulation time, OCN positive osteocytes were decreased in the 14 and 28 days. All of this may provide reasons for the decreased bone mass in the cold stimulation group.

In contrast to cold stimulation, warmth exposure has a positive effect on bone development and bone mass (Romsos et al., 1985; Serrat et al., 2008). Intestinal flora intercommunicates with host physiology, and environmental variation influences microbial composition, while the 
fluctuation of the microbiota in the gut induces changes in organ metabolism (Chevalier et al., 2015; Ziętak et al., 2016). Reports have shown that intestinal flora is an important regulator of bone metabolism (Jones et al., 2018; Li et al., 2019). Chevalier et al. have shown that transplantation of warm microbiota can protect ovariectomized mice from bone loss (Chevalier et al., 2020). It is reasonable to speculate that some changes occur in the internal microorganisms in cold-treaded mice. In addition, the gut-brainbone axis may play a role in this process (Quach and Britton, 2017).

The sympathetic nerve is considered to be an effector of bone remodeling. The sympathetic nerve is activated in a cold environment (Nedergaard and Cannon, 2014), which can decrease bone mass by regulating the balance between osteoblasts and osteoclasts (Bonnet et al., 2007; Fonseca et al., 2014; Vignaux et al., 2015). In addition, brown adipose volume is positively related to cold stimulation; and nursing mice at $4^{\circ} \mathrm{C}$ may cause increased UCP1 expression and enlarged brown adipose tissue (Cannon and Nedergaard, 2004; Bartness et al., 2010; Lim et al., 2012). Hence, the energy consumed by adipose tissue has been reported to be positively related to bone (Devlin, 2015; Lidell and Enerbäck, 2015). This might be an important factor in maintaining bone mineral density. Similarly, we confirmed BAT CM had a positive influence on the dendrites of osteocytes in this study. Qing et al. have reported that IL-6, which is mainly secreted by brown adipose tissue, is one of the most important factors under stress (Qing et al., 2020). Thus, there may be high levels of IL- 6 that led to the activation of osteoclasts in the bone loss period (Jilka et al., 1992; Lazzaro et al., 2018), but with the augmentation of brown adipose, the bone mass gets reduced.

There still have some limitations in this study. First, we attributed the fluctuation of bone mass in cold treated group to the changed osteocytes apoptosis and lacuno-canalicular area, which were not confirmed in vivo and vitro. Besides, the waved bone mass in the cold stimulated group can't be explained very well, and the reason behind it should be explored. What's more, we just use mice to detect the relationship between bone mass and temperature, there may have some difference in human.

\section{REFERENCES}

Aguirre, J. I., Plotkin, L. I., Stewart, S. A., Weinstein, R. S., Parfitt, A. M., Manolagas, S. C., et al. (2006). Osteocyte Apoptosis Is Induced by Weightlessness in Mice and Precedes Osteoclast Recruitment and Bone Loss. J. Bone Miner Res. 21 (4), 605-615. doi:10.1359/jbmr.060107

Almeida, M., Han, L., Martin-Millan, M., Plotkin, L. I., Stewart, S. A., Roberson, P. K., et al. (2007). Skeletal Involution by Age-Associated Oxidative Stress and its Acceleration by Loss of Sex Steroids. J. Biol. Chem. 282 (37), 27285-27297. doi:10.1074/jbc.m702810200

Atkins, G. J., Welldon, K. J., Wijenayaka, A. R., Bonewald, L. F., and Findlay, D. M. (2009). Vitamin K Promotes Mineralization, Osteoblast-To-Osteocyte Transition, and an Anticatabolic Phenotype by $\gamma$-carboxylation-dependent and -independent Mechanisms. Am. J. Physiology-Cell Physiol. 297 (6), C1358-C1367. doi:10.1152/ajpcell.00216.2009

Bakker, A., Klein-Nulend, J., and Burger, E. (2004). Shear Stress Inhibits while Disuse Promotes Osteocyte Apoptosis. Biochem. Biophysical Res. Commun. 320 (4), 1163-1168. doi:10.1016/j.bbrc.2004.06.056
In this study, E11, MMP13, Caspase3, RANKL TRAP and OCN expression levels change with prolonged cold exposure, which may contribute to the bone mass change during cold exposure. Low temperatures exposure induced brown adipose accumulation can influence bone mass through affecting osteocytes.

\section{DATA AVAILABILITY STATEMENT}

The raw data supporting the conclusions of this article will be made available by the authors, without undue reservation.

\section{ETHICS STATEMENT}

The animal study was reviewed and approved by the Animal Ethics Committee of Shanghai Ninth People's Hospital.

\section{AUTHOR CONTRIBUTIONS}

All authors listed have made a substantial, direct and intellectual contribution to the work, and approved it for publication.

\section{FUNDING}

This work was supported by grants from the National Natural Science Foundation of China (Nos. 11572197, 11872251, 81802679), the National Key R and D Program (grant no. 2016YFC1102100) and Natural Science Foundation of Shanghai (grant no. 20ZR1432000).

\section{ACKNOWLEDGMENTS}

We thank Dr. Lynda F. Bonewald (University of Indiana, United States) for the generous gift of the MLO-Y4 cell line.

Bartness, T. J., Vaughan, C. H., and Song, C. K. (2010). Sympathetic and Sensory Innervation of Brown Adipose Tissue. Int. J. Obes. (Lond) 34 Suppl 1 (Suppl. 10 1), S36-S42. doi:10.1038/ijo.2010.182

Bektas, A., Schurman, S. H., Sen, R., and Ferrucci, L. (2018). Aging, Inflammation and the Environment. Exp. Gerontol. 105, 10-18. doi:10.1016/ j.exger.2017.12.015

Bonewald, L. F. (2011). The Amazing Osteocyte. J. Bone Miner Res. 26 (2), 229-238. doi:10.1002/jbmr.320

Bonnet, N., Beaupied, H., Vico, L., Dolleans, E., Laroche, N., Courteix, D., et al. (2007). Combined Effects of Exercise and Propranolol on Bone Tissue in Ovariectomized Rats. J. Bone Miner Res. 22 (4), 578-588. doi:10.1359/ jbmr.070117

Cannon, B., and Nedergaard, J. (2004). Brown Adipose Tissue: Function and Physiological Significance. Physiol. Rev. 84 (1), 277-359. doi:10.1152/ physrev.00015.2003

Chen, H., Hu, B., Lv, X., Zhu, S., Zhen, G., Wan, M., et al. (2019). Prostaglandin E2 Mediates Sensory Nerve Regulation of Bone Homeostasis. Nat. Commun. 10 (1), 181. doi:10.1038/s41467-01808097-7 
Chevalier, C., Kieser, S., Çolakoğlu, M., Hadadi, N., Brun, J., Rigo, D., et al. (2020). Warmth Prevents Bone Loss Through the Gut Microbiota. Cel Metab. 32 (4), 575-590. doi:10.1016/j.cmet.2020.08.012

Chevalier, C., Stojanović, O., Colin, D. J., Suarez-Zamorano, N., Tarallo, V., VeyratDurebex, C., et al. (2015). Gut Microbiota Orchestrates Energy Homeostasis during Cold. Cell 163 (6), 1360-1374. doi:10.1016/j.cell.2015.11.004

Choy, M. H. V., Wong, R. M. Y., Chow, S. K. H., Li, M. C., Chim, Y. N., Li, T. K., et al. (2020). How Much Do We Know about the Role of Osteocytes in Different Phases of Fracture Healing? A Systematic Review. J. Orthopaedic Translation 21, 111-121. doi:10.1016/j.jot.2019.07.005

Cianciaruso, C., Beltraminelli, T., Duval, F., Nassiri, S., Hamelin, R., Mozes, A., et al. (2019). Molecular Profiling and Functional Analysis of MacrophageDerived Tumor Extracellular Vesicles. Cel Rep. 27 (10), 3062-3080. doi:10.1016/j.celrep.2019.05.008

Dallas, S. L., Prideaux, M., and Bonewald, L. F. (2013). The Osteocyte: An Endocrine Cell and More. Endocr. Rev. 34 (5), 658-690. doi:10.1210/ er.2012-1026

Devlin, M. J., Cloutier, A. M., Thomas, N. A., Panus, D. A., Lotinun, S., Pinz, I., et al. (2010). Caloric Restriction Leads to High Marrow Adiposity and Low Bone Mass in Growing Mice. J. Bone Miner Res. 25 (9), 2078-2088. doi:10.1002/ jbmr.82

Devlin, M. J. (2015). The "Skinny" on Brown Fat, Obesity, and Bone. Am. J. Phys. Anthropol. 156 (Suppl. 59), 98-115. doi:10.1002/ajpa.22661

Dolan, E. B., Haugh, M. G., Voisin, M. C., Tallon, D., and McNamara, L. M. (2015). Thermally Induced Osteocyte Damage Initiates a Remodelling Signaling cascade. PLoS One 10 (3), e0119652. doi:10.1371/journal.pone.0119652

Dole, N. S., Yee, C. S., Mazur, C. M., Acevedo, C., and Alliston, T. (2020). TGF $\beta$ Regulation of Perilacunar/Canalicular Remodeling Is Sexually Dimorphic. J. Bone Miner Res. 35 (8), 1549-1561. doi:10.1002/jbmr.4023

Emerton, K. B., Hu, B., Woo, A. A., Sinofsky, A., Hernandez, C., Majeska, R. J., et al. (2010). Osteocyte Apoptosis and Control of Bone Resorption Following Ovariectomy in Mice. Bone 46 (3), 577-583. doi:10.1016/j.bone.2009.11.006

Fonseca, T. L., Teixeira, M. B. C. G., Rodrigues-Miranda, M., Silva, M. V., Martins, G. M., Costa, C. C., et al. (2014). Thyroid Hormone Interacts with the Sympathetic Nervous System to Modulate Bone Mass and Structure in Young Adult Mice. Am. J. Physiology-Endocrinology Metab. 307 (4), E408-E418. doi:10.1152/ajpendo.00643.2013

Hadjiargyrou, M., Rightmire, E. P., Ando, T., and Lombardo, F. T. (2001). The E11 Osteoblastic Lineage Marker Is Differentially Expressed during Fracture Healing. Bone 29 (2), 149-154. doi:10.1016/s8756-3282(01)00489-6

Heimann, W. G., and Freiberger, R. H. (1960). Avascular Necrosis of the Femoral and Humeral Heads after High-Dosage Corticosteroid Therapy. N. Engl. J. Med. 263, 672-675. doi:10.1056/nejm196010062631404

Hughes, J. M., Castellani, C. M., Popp, K. L., Guerriere, K. I., Matheny, R. W., Nindl, B. C., et al. (2020). The Central Role of Osteocytes in the Four Adaptive Pathways of Bone's Mechanostat. Exerc. Sport Sci. Rev. 48 (3), 140-148. doi:10.1249/jes.0000000000000225

Ingram, J. R., Dougan, M., Rashidian, M., Knoll, M., Keliher, E. J., Garrett, S., et al. (2017). PD-L1 Is an Activation-independent Marker of Brown Adipocytes. Nat. Commun. 8 (1), 647. doi:10.1038/s41467-017-00799-8

Iolascon, G., Resmini, G., and Tarantino, U. (2013). Mechanobiology of Bone. Aging Clin. Exp. Res. 25 (Suppl. 1), S3-S7. doi:10.1007/s40520-013-0101-2

Iwaniec, U. T., Philbrick, K. A., Wong, C. P., Gordon, J. L., Kahler-Quesada, A. M., Olson, D. A., et al. (2016). Room Temperature Housing Results in Premature Cancellous Bone Loss in Growing Female Mice: Implications for the Mouse as a Preclinical Model for Age-Related Bone Loss. Osteoporos. Int. 27 (10), 3091-3101. doi:10.1007/s00198-016-3634-3

Jilka, R., Hangoc, G., Girasole, G., Passeri, G., Williams, D., Abrams, J., et al. (1992). Increased Osteoclast Development after Estrogen Loss: Mediation by Interleukin-6. Science 257 (5066), 88-91. doi:10.1126/science.1621100

Jilka, R. L., Noble, B., and Weinstein, R. S. (2013). Osteocyte Apoptosis. Bone 54 (2), 264-271. doi:10.1016/j.bone.2012.11.038

Jones, R. M., Mulle, J. G., and Pacifici, R. (2018). Osteomicrobiology: The Influence of Gut Microbiota on Bone in Health and Disease. Bone 115, 59-67. doi:10.1016/j.bone.2017.04.009

Jung, S. M., Sanchez-Gurmaches, J., and Guertin, D. A. (2019). Brown Adipose Tissue Development and Metabolism. Handb Exp. Pharmacol. 251, 3-36. doi:10.1007/164_2018_168
Kegelman, C. D., Coulombe, J. C., Jordan, K. M., Horan, D. J., Qin, L., Robling, A. G., et al. (2020). YAP and TAZ Mediate Osteocyte Perilacunar/Canalicular Remodeling. J. Bone Miner Res. 35 (1), 196-210. doi:10.1002/jbmr.3876

Kennedy, O. D., Herman, B. C., Laudier, D. M., Majeska, R. J., Sun, H. B., and Schaffler, M. B. (2012). Activation of Resorption in Fatigue-Loaded Bone Involves Both Apoptosis and Active Pro-osteoclastogenic Signaling by Distinct Osteocyte Populations. Bone 50 (5), 1115-1122. doi:10.1016/ j.bone.2012.01.025

Komori, T. (2013). Functions of the Osteocyte Network in the Regulation of Bone Mass. Cell Tissue Res 352 (2), 191-198. doi:10.1007/s00441-012-1546-x

Lazzaro, L., Tonkin, B. A., Poulton, I. J., McGregor, N. E., Ferlin, W., and Sims, N. A. (2018). IL-6 Trans -signalling Mediates Trabecular, but Not Cortical, Bone Loss after Ovariectomy. Bone 112, 120-127. doi:10.1016/j.bone.2018.04.015

Li, L., Rao, S., Cheng, Y., Zhuo, X., Deng, C., Xu, N., et al. (2019). Microbial Osteoporosis: The Interplay between the Gut Microbiota and Bones via Host Metabolism and Immunity. Microbiologyopen 8 (8), e00810. doi:10.1002/ mbo3.810

Lidell, M. E., and Enerbäck, S. (2015). Brown Adipose Tissue and Bone. Int. J. Obes. Suppl. 5 (Suppl. 1), S23-S27. doi:10.1038/ijosup.2015.7

Lim, S., Honek, J., Xue, Y., Seki, T., Cao, Z., Andersson, P., et al. (2012). Coldinduced Activation of Brown Adipose Tissue and Adipose Angiogenesis in Mice. Nat. Protoc. 7 (3), 606-615. doi:10.1038/nprot.2012.013

Marahleh, A., Kitaura, H., Ohori, F., Kishikawa, A., Ogawa, S., Shen, W.-R., et al. (2019). TNF- $\alpha$ Directly Enhances Osteocyte RANKL Expression and Promotes Osteoclast Formation. Front. Immunol. 10, 2925. doi:10.3389/ fimmu.2019.02925

Mazur, C. M., Woo, J. J., Yee, C. S., Fields, A. J., Acevedo, C., Bailey, K. N., et al. (2019). Osteocyte Dysfunction Promotes Osteoarthritis through MMP13dependent Suppression of Subchondral Bone Homeostasis. Bone Res. 7, 34. doi:10.1038/s41413-019-0070-y

Moriishi, T., Fukuyama, R., Ito, M., Miyazaki, T., Maeno, T., Kawai, Y., et al. (2012). Osteocyte Network; a Negative Regulatory System for Bone Mass Augmented by the Induction of Rankl in Osteoblasts and Sost in Osteocytes at Unloading. PLoS One 7 (6), e40143. doi:10.1371/journal.pone.0040143

Nakashima, T., Hayashi, M., Fukunaga, T., Kurata, K., Oh-hora, M., Feng, J. Q., et al. (2011). Evidence for Osteocyte Regulation of Bone Homeostasis through RANKL Expression. Nat. Med. 17 (10), 1231-1234. doi:10.1038/nm.2452

Nedergaard, J., and Cannon, B. (2014). The browning of white Adipose Tissue: Some Burning Issues. Cel Metab. 20 (3), 396-407. doi:10.1016/ j.cmet.2014.07.005

Nicks, K. M., Amin, S., Atkinson, E. J., Riggs, B. L., Melton, L. J., and Khosla, S. (2012). Relationship of Age to Bone Microstructure Independent of Areal Bone mineral Density. J. Bone Miner Res. 27 (3), 637-644. doi:10.1002/jbmr.1468

Nose, K., Saito, H., and Kuroki, T. (1990). Isolation of a Gene Sequence Induced Later by Tumor-Promoting 12-O-Tetradecanoylphorbol-13-Acetate in Mouse Osteoblastic Cells (MC3T3-E1) and Expressed Constitutively in RasTransformed Cells. Cell Growth Differ 1 (11), 511-518.

Palermo, A., Tuccinardi, D., D’Onofrio, L., Watanabe, M., Maggi, D., Maurizi, A. R., et al. (2017). Vitamin K and Osteoporosis: Myth or Reality. Metabolism 70, 57-71. doi:10.1016/j.metabol.2017.01.032

Qin, L., Liu, W., Cao, H., and Xiao, G. (2020). Molecular Mechanosensors in Osteocytes. Bone Res. 8 (1), 23. doi:10.1038/s41413-020-0099-y

Qing, H., Ardeshirpour, L., Divieti Pajevic, P., Dusevich, V., Jähn, K., Kato, S., et al. (2012). Demonstration of Osteocytic Perilacunar/canalicular Remodeling in Mice during Lactation. J. Bone Miner Res. 27 (5), 1018-1029. doi:10.1002/ jbmr.1567

Qing, H., Desrouleaux, R., Israni-Winger, K., Mineur, Y. S., Fogelman, N., Zhang, C., et al. (2020). Origin and Function of Stress-Induced IL-6 in Murine Models. Cell 182 (2), 372-387. doi:10.1016/j.cell.2020.05.054

Quach, D., and Britton, R. A. (2017). Gut Microbiota and Bone Health. Adv. Exp. Med. Biol. 1033, 47-58. doi:10.1007/978-3-319-66653-2_4

Robbins, A., Tom, C. A. T. M. B., Cosman, M. N., Moursi, C., Shipp, L., Spencer, T. M., et al. (2018). Low Temperature Decreases Bone Mass in Mice: Implications for Humans. Am. J. Phys. Anthropol. 167 (3), 557-568. doi:10.1002/ajpa.23684

Romsos, D. R., Ferguson, D., and Vander Tuig, J. G. (1985). Effects of a Warm Environment on Energy Balance in Obese () Mice. Metabolism 34 (10), 931-937. doi:10.1016/0026-0495(85)90141-6 
Schacht, V., Ramirez, M., Hong, Y. K., Hirakawa, S., Feng, D., Harvey, N., et al. (2003). T1alpha/podoplanin Deficiency Disrupts normal Lymphatic Vasculature Formation and Causes Lymphedema. EMBO J. 22 (14), 3546-3556. doi:10.1093/emboj/cdg342

Serrat, M. A., King, D., and Lovejoy, C. O. (2008). Temperature Regulates Limb Length in Homeotherms by Directly Modulating Cartilage Growth. Proc. Natl. Acad. Sci. 105 (49), 19348-19353. doi:10.1073/pnas.0803319105

Shi, Y., Yadav, V. K., Suda, N., Liu, X. S., Guo, X. E., Myers, M. G., et al. (2008). Dissociation of the Neuronal Regulation of Bone Mass and Energy Metabolism by Leptin In Vivo. Proc. Natl. Acad. Sci. 105 (51), 20529-20533. doi:10.1073/pnas.0808701106

Silva, R. A. B., Sousa-Pereira, A. P., Lucisano, M. P., Romualdo, P. C., Paula-Silva, F. W. G., Consolaro, A., et al. (2020). Alendronate Inhibits Osteocyte Apoptosis and Inflammation via IL -6, Inhibiting Bone Resorption in Periapical Lesions of Ovariectomized Rats. Int. Endod. J. 53 (1), 84-96. doi:10.1111/iej.13206

Song, L., Tang, S., Han, X., Jiang, Z., Dong, L., Liu, C., et al. (2019). KIBRA Controls Exosome Secretion via Inhibiting the Proteasomal Degradation of Rab27a. Nat. Commun. 10 (1), 1639. doi:10.1038/s41467-019-09720-x

Staines, K. A., Ikpegbu, E., Törnqvist, A. E., Dillon, S., Javaheri, B., Amin, A. K., et al. (2019). Conditional Deletion of E11/podoplanin in Bone Protects against Load-Induced Osteoarthritis. BMC Musculoskelet. Disord. 20 (1), 344. doi:10.1186/s12891-019-2731-9

Steinberg, B., Singh, I. J., and Mitchell, O. G. (1981). The Effects of Cold-Stress, Hibernation, and Prolonged Inactivity on Bone Dynamics in the golden Hamster,Mesocricetus auratus. J. Morphol. 167 (1), 43-51. doi:10.1002/ jmor.1051670105

Tang, S. Y., Herber, R.-P., Ho, S. P., and Alliston, T. (2012). Matrix Metalloproteinase-13 Is Required for Osteocytic Perilacunar Remodeling and Maintains Bone Fracture Resistance. J. Bone Miner Res. 27 (9), 1936-1950. doi:10.1002/jbmr.1646

Uda, Y., Azab, E., Sun, N., Shi, C., and Pajevic, P. D. (2017). Osteocyte Mechanobiology. Curr. Osteoporos. Rep. 15 (4), 318-325. doi:10.1007/ s11914-017-0373-0

Vignaux, G., Ndong, J. D., Perrien, D. S., and Elefteriou, F. (2015). Inner Ear Vestibular Signals Regulate Bone Remodeling via the Sympathetic Nervous System. J. Bone Miner Res. 30 (6), 1103-1111. doi:10.1002/jbmr.2426

Wang, L., Ciani, C., Doty, S. B., and Fritton, S. P. (2004). Delineating Bone's Interstitial Fluid Pathway In Vivo. Bone 34 (3), 499-509. doi:10.1016/ j.bone.2003.11.022

Wang, L. (2018). Solute Transport in the Bone Lacunar-Canalicular System (LCS). Curr. Osteoporos. Rep. 16 (1), 32-41. doi:10.1007/s11914-018-0414-3

Wee, N. K. Y., Nguyen, A. D., Enriquez, R. F., Zhang, L., Herzog, H., and Baldock, P. A. (2020). Neuropeptide Y Regulation of Energy Partitioning and Bone Mass During Cold Exposure. Calcif Tissue Int. 107 (5), 510-523. doi:10.1007/s00223-020-00745-9
Weinbaum, S., Cowin, S. C., and Zeng, Y. (1994). A Model for the Excitation of Osteocytes by Mechanical Loading-Induced Bone Fluid Shear Stresses. J. Biomech. 27 (3), 339-360. doi:10.1016/0021-9290(94)90010-8

Wetterwald, A., Hofstetter, W., Cecchini, M. G., Lanske, B., Wagner, C., Fleisch, H., et al. (1996). Characterization and Cloning of the E11 Antigen, a Marker Expressed by Rat Osteoblasts and Osteocytes. Bone 18 (2), 125-132. doi:10.1016/8756-3282(95)00457-2

Wu, Q., Zhou, X., Huang, D., Ji, Y., and Kang, F. (2017). IL-6 Enhances OsteocyteMediated Osteoclastogenesis by Promoting JAK2 and RANKL Activity In Vitro. Cell Physiol Biochem 41 (4), 1360-1369. doi:10.1159/000465455

Wysolmerski, J. J. (2013). Osteocytes Remove and Replace Perilacunar mineral during Reproductive Cycles. Bone 54 (2), 230-236. doi:10.1016/ j.bone.2013.01.025

Zhang, K., Barragan-Adjemian, C., Ye, L., Kotha, S., Dallas, M., Lu, Y., et al. (2006). E11/gp38 Selective Expression in Osteocytes: Regulation by Mechanical Strain and Role in Dendrite Elongation. Mol. Cel Biol 26 (12), 4539-4552. doi:10.1128/ mcb.02120-05

Zhou, F., Mei, J., Yuan, K., Han, X., Qiao, H., and Tang, T. (2019). Isorhamnetin Attenuates Osteoarthritis by Inhibiting Osteoclastogenesis and Protecting Chondrocytes through Modulating Reactive Oxygen Species Homeostasis. J. Cel Mol Med 23 (6), 4395-4407. doi:10.1111/jcmm.14333

Ziętak, M., Kovatcheva-Datchary, P., Markiewicz, L. H., Ståhlman, M., Kozak, L. P., and Bäckhed, F. (2016). Altered Microbiota Contributes to Reduced DietInduced Obesity upon Cold Exposure. Cell Metab 23 (6), 1216-1223. doi:10.1016/j.cmet.2016.05.001

Conflict of Interest: The authors declare that the research was conducted in the absence of any commercial or financial relationships that could be construed as a potential conflict of interest.

Publisher's Note: All claims expressed in this article are solely those of the authors and do not necessarily represent those of their affiliated organizations, or those of the publisher, the editors and the reviewers. Any product that may be evaluated in this article, or claim that may be made by its manufacturer, is not guaranteed or endorsed by the publisher.

Copyright $\odot 2021 \mathrm{Du}, \mathrm{He}, \mathrm{Cui}, \mathrm{Li}, \mathrm{Xu}$, Zhang, Zhang, Yan, Qu and Yu. This is an open-access article distributed under the terms of the Creative Commons Attribution License (CC BY). The use, distribution or reproduction in other forums is permitted, provided the original author(s) and the copyright owner(s) are credited and that the original publication in this journal is cited, in accordance with accepted academic practice. No use, distribution or reproduction is permitted which does not comply with these terms. 